\title{
O Estudo de Caso Naturalista na investigação de Arranjos Produtivos Locais (APL) de Tecnologias de Informação e Comunicação (TIC)
}

\author{
Anderson Diego Farias da SILVA \\ Universidade Federal de Pernambuco (UFPE), Recife, PE, Brasil
}

Nelson da Cruz Monteiro FERNANDES

Universidade Federal de Pernambuco (UFPE), Caruaru, PE, Brasil

Fernando Gomes de PAIVA JÚNIOR

Universidade Federal de Pernambuco (UFPE), Recife, PE, Brasil

Received 22 out. 17; Accepted 10 nov. 17
Evaluation System: Guest Article
Editor: Jose Lindenberg Julião Xavier Filho, Dr.
ISSN: 2594-8040

To cite this paper: Silva, A. D. F.; Fernandes, N. C. M. \& Paiva Júnior, F. G. (2017). O Estudo de Caso Naturalista na investigação de Arranjos Produtivos Locais (APL) de Tecnologias de Informação e Comunicação (TIC). Journal of Perspectives in Management - JPM, 1(1), p. 36-50.

\section{Resumo}

O Estudo de Caso (EC) envolve um cenário conceitual divergente podendo ser utilizado em formas de pesquisa diversificadas no campo dos estudos organizacionais com respeito ao valor e à plausibilidade da aplicação desse método de investigação em Arranjos Produtivos Locais (APL). Neste ensaio teórico-metodológico, discutimos a interpelação conceitual do Estudo de Caso naturalista de Robert Stake em meio as suas possibilidades e limitações de uso, ilustradas por meio da análise do Arranjo Produtivo Local (APL) de Tecnologias de Informação e Comunicação (TIC) e Economia Criativa do Estado de Pernambuco (Brasil), denominado Porto Digital. Ao migrar de uma abordagem fundamentada na lógica da explicação de perspectiva funcionalista de Yin (2005) para o tratamento de Stake (2005) essa visão de estudo de caso naturalista representa a oportunidade para o desenvolvimento de estudos interpretativos que possibilitam o incremento do conhecimento relacionado à ambientes de negócios cooperativos contemplando as novas TIC.

Palavras-Chave: Abordagens Metodológicas Qualitativas; Estudos de Casos; Arranjos Produtivos Locais (APL), tecnologia de Informação e Comunicação (TIC). 


\section{Introdução}

O estudo de caso (EC) tem alcançado popularidade em pesquisas desenvolvidas no campo dos estudos organizacionais, conforme podemos atestar nos estudos de Célia e Loiola (2014), Senger, Paço-Cunha e Senger (2013), Langley e Royer (2006), Heracleous e Barrett (2001), Hartley (1995) e Biggart (1977). Esse fenômeno é explicado, segundo Cohen e Manion (1989) e Aires (2011), pela relativização da investigação desenvolvida sob o paradigma positivista por parte da comunidade de investigadores em estudos organizacionais, levando ao desenvolvimento do estudo de caso como algo parecido a uma indústria em expansão (Hancock \& Algozzine, 2016; Coutinho \& Chaves, 2002; Bryman, 1992; Hassard, 1991; Cohen \& Manion, 1989).

Os investigadores de arranjos produtivos de TIC não ficaram alheios a esse interesse crescente pelo EC. Isso reflete uma inflexão paradigmática que vem influenciando a utilização de novas abordagens metodológicas que auxiliam na investigação de problemáticas complexas no campo dos estudos organizacionais. A isso, soma-se o interesse crescente dos pesquisadores dessas áreas que se debruçam para analisar organizações ou grupos com os quais possuam vínculos cooperativos, ou seja, estão imersos no interior desses arranjos produtivos.

Os estudos de Godoi e Balsini (2010) demonstraram que o EC tem sido bastante utilizado nas pesquisas no campo dos estudos organizacionais. Mariz, Silva, Dourado e Regis (2005) e Godoi e Balsini (2010) realizaram pesquisas documentais em anais de eventos realizados no Brasil, bem como em periódicos brasileiros, e constataram um expressivo número de trabalhos utilizando esse tipo de método. Entretanto, sem entrar no mérito de utilização do

\section{Abordagens Qualitativas na investigação de Fenômenos Emergentes nos Estudos Organizacionais}

Pesquisas recentes realizadas por Santos (2017), Leão, Mello e Vieira (2009), Paiva Júnior e Mello (2008) e Santos (2006), sugerem que a questão da cientificidade e as perspectivas paradigmáticas com suas implicações nos métodos de pesquisa vêm sendo alvos de debates, cujo epicentro encontra sua gênese em estudos como o elaborado por Burrel e Morgan (1979). Este, além de contemplar a teoria organizacional como lócus de investigação específico trazem à tona a teoria social tratada de método, observamos que a grande maioria desses estudos está alinhada à perspectiva de EC desenvolvida por Yin (2005). A possível causa desta opção reside no fato de essa abordagem está alinhada a uma característica mais funcionalista como perspectiva paradigmática ainda dominante no campo dos estudos organizacionais.

Num esforço por se empreender um novo olhar sobre o EC, este trabalho apresenta a abordagem naturalista do EC sob a perspectiva proposta por Stake e Easley (1979) e Stake (2006; 2005; 2000; 1988), na qual existe certa potencialidade para o estudo ser aplicado para situações diversas de investigação, inclusive no que tange ao domínio de fenômenos que acontecem no bojo de arranjos produtivos de TIC.

Ao analisarmos as obras de Robert E. Stake se reconhece o cenário de que, ao contrário do que preconizam a maioria dos manuais de métodos qualitativos, não existe uma estrutura padronizada ou pré-definida na operacionalização desta técnica de investigação. Portanto, a análise do estudo de caso que apresentamos parte de uma estrutura desenvolvida a partir de tópicos das obras de Stake e Easley (1979) e Stake (2006; 2005; 2000; 1988). A pouca tradição desta metodologia na investigação sobre arranjos produtivos de TIC no Brasil, associada ao fato de o EC naturalista ser uma abordagem metodológica exigente, que pode conduzir a resultados valiosos como a absolutamente estéreis, justificam a pertinência do presente estudo e ensejam a seguinte questão norteadora: Como o método de Estudo de Caso pautado na perspectiva naturalista auxilia na investigação a respeito de Arranjos Produtivos de TIC?

modo mais ampla. Logo, autores como Bauer e Gaskell (2002), Lewis e Grimes (1999), Schultz e Hatch, (1996) e Goia e Pitre (1990), vêm difundindo a perspectiva multiparadigmática, uma vez que apresenta determinadas posições metateóricas relacionadas a diferentes paradigmas presentes no âmago das tradições de pesquisa vigentes (Godoi \& Balsini, 2010; Silva \& Neto, 2006).

$\mathrm{O}$ interesse pelo uso de abordagens metodológicas de cunho qualitativo em investigações efetuadas no campo dos estudos organizacionais surge a partir dos anos 1960, em contraposição à predominância de abordagens subordinadas ao positivismo (Santos, 2017; Godoi \& Balsini, 2010; Rocha \& Ceretta, 1998). 
Não obstante, o crescente uso de abordagens metodológicas qualitativas (Lima, Maia, Soares \& Ipiranga, 2002), persistem "controvérsias sobre a natureza, os critérios de adequação metodológica e os parâmetros de avaliação desse tipo de pesquisa" (Godoi \& Balsini, 2010, p. 90). Os debates que permeiam o rigor científico e as características peculiares de pesquisas no âmbito dos estudos organizacionais realizadas por meio de abordagens qualitativas têm despertado o surgimento de estudos de natureza crítica que buscam tratar dessa fragilidade epistemometodológicas encontrada em estudos de cunho qualitativo (Paiva Júnior, Leão \& Mello, 2011).

A discussão da cientificidade na pesquisa qualitativa se vincula à necessidade de aprimoramento dos métodos qualitativos utilizados em estudos organizacionais, compreendendo que o científico não é o que foi verificado, mas o que se mantém discutível, conforme lembram Demo (2001) e Martinelli (1999). Com respeito a isso, os estudos de Pinto e Santos (2008) e Godoy (1995) revelam que já na década de 1970, uma publicação da revista Administrative Science Quarterly foi dedicada ao tema "Qualitative Methodology" e nas décadas subsequentes, o enfoque qualitativo foi sendo usado de forma cada vez mais frequente em pesquisas realizadas no domínio dos estudos organizacionais, ocasionando relevantes contribuições para a Área como podem ser verificados com Sebring (1977), Hirsch (1975), Lawrence e Lorsch (1967), todos publicados na Administrative Science Quarterly.

$\mathrm{Na}$ sessão a seguir, nos aprofundaremos sobre o conceito de Estudo de Caso, seus principais teóricos, a perspectiva do estudo de caso naturalista e o modelo teórico que exemplifica as tipologias do estudo de caso na perspectiva naturalista.

\subsection{O que é um Estudo de Caso?}

O estudo de caso é um método de pesquisa há muito utilizado no campo dos estudos organizacionais. A pesquisa neste campo tem como foco central as organizações ou grupos a elas relacionados. Portanto, o ato de estudá-las como "casos" parece ser uma estratégia metodológica relacionada à construção e ao acumulo de conhecimento, conforme argumentam Leão, Vieira, Gaião e Souza (2012). Logo, essa concepção corrobora com os argumentos de Goode e Hatt (1968, p. 421), que definem o estudo de caso como sendo um:
[...] meio de organizar os dados sociais preservando o caráter unitário do objeto social estudado [...] uma abordagem que considera qualquer unidade social como um todo [...] [incluindo] o desenvolvimento dessa unidade, que pode ser uma pessoa, uma família ou outro grupo social, um conjunto de relações ou processos (como crises familiares, ajustamento à doença, formação de amizade, invasão étnica de uma vizinhança etc.) ou mesmo toda uma cultura [...].

Os autores caracterizam o estudo de caso como um "tipo de questão [...] cujo foco de interesse está no individual, no especifico" (Godoi \& Balsini, 2010, p. 119). Essa visão de especificidade episódica do caso é compartilhada pelos estudos de Abma e Stake (2014), Stake (2005; 2000; 1988), Yin (2005), Eisenhardt (1989) e Merriam (1998; 1988).

A principal característica do EC em relação a outras modalidades de pesquisas compreende, de acordo com Stake (1988, p. 256), "a busca do pesquisador por uma compreensão de um caso particular, em sua idiossincrasia, em sua complexidade". Um segundo aspecto, ainda segundo o autor, está relacionado ao momento em que nos referimos ao estudo de caso, estamos buscando descrever um método de pesquisa a partir de opções por determinados objetos escolhidos para serem estudados, que correspondem a uma pessoa, uma instituição, uma empresa, um programa ou certo grupo de pessoas que compartilhem de uma determinada experiência semelhante ou mesmo um ambiente específico. Merriam (1998; 1988) corrobora com esse argumento e acrescenta que os pesquisadores que optam pelo EC almejam compreender uma determinada situação de forma mais aprofundada, priorizando o seu significado tratado de forma múltipla para os envolvidos.

Já para Yin (2005) numa definição mais técnica do EC, devem ser levados em conta dois aspectos relevantes, que são o escopo e o componente metodológico. Quanto ao escopo, Yin (2005, p. 32) entende que

Um estudo de caso é uma investigação empírica que investiga um fenômeno contemporâneo dentro de seu contexto da vida real, especialmente quando os limites entre o fenômeno e o contexto não estão claramente definidos.

Já em relação aos componentes metodológicos, Yin (2005, p. 32-33) garante que

a investigação de estudo de caso enfrenta uma situação tecnicamente única em que haverá muito mais variáveis de interesse do que pontos de dados, e, como resultado, baseia-se em várias fontes de evidência, com os dados precisando convergir em um formato de triângulo e, 
como outro resultado, beneficia-se do desenvolvimento prévio de proposições teóricas para conduzir a coleta e a análise dos dados.

Existe certa concordância entre os especialistas em formulação de teorias sobre EC com relação ao primeiro aspecto trazido por Yin (2005), entretanto, o segundo aspecto, o metodológico, constitui motivo de debates relativos à manutenção de um diálogo profícuo para a área, oriundo de pontos de vista distintos. Acerca dessas particularidades referentes aos aspectos metodológicos do estudo caso, podemos citar o argumento de Bell (1989) que o define como um termo-chave, contemplando um conjunto de métodos de pesquisas voltados para a compreensão de interações sistêmicas entre fatores e eventos. Fidel (1992) concebe o EC como um método específico de se produzir uma pesquisa de campo. Para esse autor, estas pesquisas de campo deverão averiguar os fenômenos e o modo como estes ocorrem, no entanto, sem interferências do investigador. Por fim, podemos citar o argumento de Ponte (2006, p. 2) que afirma que o EC

É uma investigação que se assume como particularística, isto é, que se debruça deliberadamente sobre uma situação específica que se supõe ser única ou especial, pelo menos em certos aspectos, procurando descobrir o que há nela de mais essencial e característico e, desse modo, contribuir para a compreensão global de um certo fenômeno de interesse.

Porém, não será nosso objetivo no presente estudo nos aprofundarmos as diversas perspectivas dos métodos de EC, acreditamos que esta poderá ser uma questão para estudos futuros. Entretanto, a seguir apresentaremos a discussão sobre o estudo de caso na perspectiva naturalista de Stake e Easley (1979) e Stake $(2006 ; 2005 ; 2000 ; 1988)$.

\subsection{Estudo de Caso Naturalista}

As pesquisas referentes ao uso do método de estudo de caso na concepção de Robert Stake são desenvolvidas em sua área de pesquisa, a educação, no final dos anos 1970 (Stake \& Easley, 1979), passando nos anos seguintes a ganhar força no campo das ciências sociais, a partir da publicação das obras "Case Studies" e "Qualitative Case Studies", ambas editadas no Handbook of Qualitative Research, publicado por Denzin e Lincoln (2005).

$\mathrm{Na}$ perspectiva de Stake (2000), um EC bem formulado consiste em oferecer reflexão e a aquisição de novos conhecimentos tanto para o pesquisador quanto para o campo de estudo. A visão naturalista de estudo de caso proporcionada por este autor pode gerar um conhecimento vicário para aqueles que querem lançar mão das suas contribuições epistemológicas. Essa perspectiva de estudo permite à amplificação de um determinado conhecimento, permitindo aos pesquisadores a compreensão de outros casos e diferentemente da perspectiva de estudo de caso funcionalista, que possui forte influência da abordagem quantitativa, onde assumem uma ontologia realista e se enquadram na perspectiva paradigmática positivista (Leão et al., 2012).

A abordagem de EC formulada por Stake (2000) está vinculada a perspectiva paradigmática interpretativista. Nesse sentido, o pesquisador que adota este método de investigação deverá desenvolvê-lo por meio da inserção de um investigador no campo de pesquisa, no qual deverá observar e registrar o caso estudado (Leão et al., 2012).

A seguir, estão as categorias de análise inspirada no método de EC de Stake (2000) e que nos auxiliarão na apreciação do caso ilustrativo a ser investigado nas próximas sessões.

Inicialmente, com relação ao trabalho de campo, algumas questões precisarão ser observadas pelo pesquisador. Para tanto, Stake (2000; 1988) apresenta algumas preocupações, tais como a seleção do caso ou dos casos, bem como o acesso aos mesmos; a interatividade do caso com o seu contexto; a equipe de pesquisadores que conduzem o estudo, especialmente no uso de estudos de caso múltiplos; além de questões éticas que deverão ser analisadas.

Em relação à seleção do(s) caso(s), deverá ser levada em consideração a representatividade do fenômeno a ser estudado, se o mesmo permitirá um acumulo de conhecimento e se existirá acesso para o desenvolvimento do trabalho (Stake, 2006; 2005).

Em se tratando da interatividade com o contexto, Stake (2005) propõe que o EC qualitativo deverá procurar apreender acerca da complexidade existente na relação de interação que ocorre nas mais variadas situações, como no caso das empresas imersas em redes dinâmicas de negócios, das quais dificilmente poderiam ser entendidas nos estudos de cunho quantitativo.

A busca pela transferência de uma representação fidedigna das experiências do pesquisador constitui uma das observações mais frequentes da visão de EC difundida por Stake (2006), especialmente, com relação aos do tipo multicasos. Por isso, o autor apresenta o trabalho experiente de uma equipe como um fator que auxilia no desenvolvimento pleno de pesquisas orientadas por essa estratégia de pesquisa. 
Igualmente, ainda em consideração ao trabalho de campo, se observa que as vinculações das questões éticas estão relacionadas com o acesso a informações, exposições das pessoas e interpretações coerentes dessas informações (Stake, 2005).

Relativamente à coleta de dados, os estudos de cunho naturalista privilegiam a observação como método que propicia um melhor entendimento do caso (Lincoln \& Guba, 1985). Assim, na maioria dos EC (figura 1), o estabelecimento de uma relação entre uma dada situação e seu contexto geralmente é realizado por meio da aplicação dos métodos observacionais diretos ou indiretos de coleta de dados.

O método da observação direta permite uma aproximação maior do pesquisador com o contexto do objeto em estudo e, como tal, ajudam na identificação dos fatores que corroboram para o entendimento do caso. O método da observação indireta poderá ser adotado quando inexistir a possibilidade do pesquisador estar presente na situação. Nesse caso, os dados serão obtidos por meio da investigação junto a indivíduos presentes na atividade estudada (Abma \& Stake, 2014; Stake, 2006). Além disso, outro método de coleta de dados bastante significativo para os EC qualitativos que são as entrevistas, pois, tal método permite ao pesquisador uma visão mais abrangente dos acontecimentos que não puderam ser presenciados (Stake, 1995).

Ademais, o pesquisador poderá acessar documentos oficiais, relatórios e demais publicações a fim de se encontrar dados que contribuam para uma melhor observação do caso estudado. A pesquisa em registros é válida, pois, por meio dela poderão ser obtidos informações e detalhes importantes para o estudo realizado (Stake, 1995), além de trazer maior legitimidade aos dados, maior qualidade das informações e aumentar a experiência vivida pelo pesquisador, que desse modo tem melhores condições de significar as evidencias que permitem considerar as razões que levam os pesquisados a pensar e sentir o fenômeno estudado de determinadas maneiras

No processo de interpretação poderão ser aplicados procedimentos de triangulação e validação, que têm como objetivo eliminar possíveis interpretações equivocadas do caso, conforme assinalam Gaskell e Bauer (2002) e Abma e Stake (2014), Stake (1999). As tipologias nos procedimentos de triangulação que estão disponíveis ao pesquisador versam sobre a triangulação de fontes de dados, na utilização de diversas fontes de informação; e a triangulação de investigadores, que sugere que mais de um pesquisador possa analisar os mesmos dados (Denzin, 2009).

\subsubsection{Tipologia do Estudo de Caso Naturalista}

A definição do tipo de estudo de caso a ser aplicada ocorre por meio da escolha do número de casos a serem analisados, assim como, no enfoque que será empreendido pelo estudo. Na figura 1, desenvolvida por Leão et al. (2012), podemos observar a tipologia do estudo de caso naturalista, no qual concebe o estudo de caso único; estudo de multicaso; estudo de caso intrínseco; e o estudo de caso instrumental.

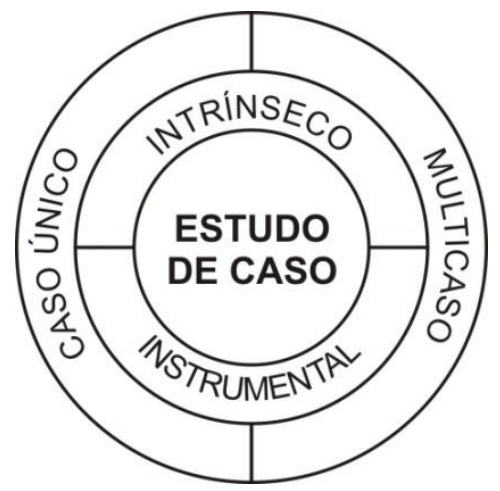

Figura 1. Tipologia do estudo de caso naturalista Fonte: Leão et al. (2012)

O estudo de caso único, é compreendido como sendo aquele em que o pesquisador se dedica por analisar um fenômeno específico e singular, dentro de seu contexto específico. Com relação ao estudo de multicasos, é descrito como sendo aquele em que se permite estudar diversos casos individuais desde que possuam uma correlação para a compreensão do fenômeno estudado como um todo.

No tipo de estudo EC intrínseco existe uma intenção em se aprofundar sobre cada uma das especificidades presentes no caso estudado. Nesse sentido, Meirinhos e Osório (2010, p. 58) corroboram com Leão et al. (2012), afirmando que, em relação ao EC intrínseco, "o importante é compreender exclusivamente o caso particular, sem relação com outros casos ou outras problemáticas mais abrangentes". Por fim, a perspectiva de EC instrumental proposta Stake (2000), vislumbra atender ao tipo de pesquisa em que se excedem as limitações do caso em si, o que irá possibilitar por meio da singularidade deste atingir um entendimento geral. Meirinhos e Osório (2010) afirmam que este tipo de $\mathrm{EC}$ em si tem um interesse que tenderá a ser mais secundário e se distinguem dos tipos de EC intrínsecos porque se caracterizam pelo que desejam 
conhecer e compreender de uma determinada problemática mais ampla, por meio da compreensão do caso particular.

$\mathrm{Na}$ sessão a seguir, iremos apresentar por meio das categorias analíticas de um EC Naturalista destacadas anteriormente, como podemos aplicar esta modalidade de EC desenvolvida por Stake (2005; 2000) em investigações sobre APL de TIC e Economia Criativa.

\section{O Estudo de Caso Naturalista na} investigação do Arranjo Produtivo Local de TIC e Economia Criativa de Recife-PE

O EC que optamos por ilustrar e aplicar a nossa pesquisa corresponde ao Arranjo Produtivo Local urbano de Tecnologia da Informação e Comunicação (TIC) e Economia Criativa, denominado Porto Digital (PD), localizado na Cidade Metropolitana do Recife, no Nordeste brasileiro. Este cluster é reconhecido por articular agentes empreendedores em uma rede colaborativa complexa por meio de um modelo de governança que envolve entes vinculados as esferas público-privada e que visam à produção de bens simbólicos (Porto Digital, 2015; Paiva Júnior, Corrêa \& Souza, 2006).

\subsection{A seleção do Caso}

Em dezessete anos de atuação, o Porto Digital se consolida como um dos principais Arranjos Produtivos Locais (APL) de Tecnologia do país, conforme demonstrado pelo relatório da Consultoria Internacional McKinsey Global Institute (2011). Destacam-se os investimentos realizados na melhoria dos processos de desenvolvimento de software, que propiciam a instalação de centenas de empresas advindas da Região Metropolitana do Recife, de outros estados e países (Silva, 2014; , Fernandes, Silva \& Paiva Júnior, 2012).

O Porto Digital surgiu da articulação de caráter empreendedor de vários atores, entre eles: o governo do Estado de Pernambuco, o Centro de Estudos e Sistemas Avançados do Recife - C.E.S.A.R, o Centro de Informática da Universidade Federal de Pernambuco CIn, e outros órgãos e empresas privadas do setor de tecnologia da região visando o desenvolvimento econômico e social do Estado de Pernambuco e tendo como ferramenta o setor da Tecnologia da Informação e Comunicação (TIC) (Paiva Júnior et al., 2006, p. 8).

O cluster é composto, em sua maioria, por pequenas e médias empresas, entretanto, empreendimentos multinacionais como a Motorola, IBM, Samsung e Microsoft também estão inseridas no polo. Atualmente, esse APL envolve 230 empresas, representadas por meio das empresas de softwares e serviços de TIC, empresas incubadas, empresas de economia criativa e empresas que prestam serviços às empresas de TIC. Além disso, de acordo com informações recentes do seu portal institucional, o arranjo conta com 7.055 colaboradores, sendo que desses, $75 \%$ possuem o Ensino Superior (Porto Digital, 2015; Silva, 2014).

Desde a sua constituição, o PD estabelece compromissos visando à revitalização do núcleo urbano recifense, sendo considerada intencional a sua escolha pelo bairro do Recife, pelo seu valor simbólico. Buscando associar o passado histórico de prosperidade da Cidade ao novo ambiente de negócios que se estabelece, ao mesmo tempo, Governo, Empresas e Entidades para-estatais, constituíram um modelo de gestão triple helix (Etzkowitz \& Leydsdorff, 1997), no intuito de desenvolver um ambiente para o desenvolvimento de negócios inseridos na dinâmica da economia do conhecimento.

Com relação ao faturamento produzido pelo arranjo, no ano de 2010, o Porto Digital obteve um faturamento: R\$ 870.873.585,00. Em 2012, o cluster divulgou uma nova pesquisa desenvolvida pela agência Datamétrica, onde registrou um faturamento de R\$1.000.528.105,39 (Porto Digital, 2015). A gestão do APL é efetuada como Organização Social (OS) denominada de Núcleo de Gestão do Porto Digital (NGPD), que recuperou edifícios para instalar as principais âncoras que trabalham em parceria. Seus objetivos, além da administração do parque tecnológico, buscam atrair investimentos e negócios. Além disso, o NGPD atua no sentido de criar mercados para o desenvolvimento de pesquisas tecnológicas; promover a aproximação entre o meio acadêmico e o empresarial; apoiar e incentivar o desenvolvimento de novos produtos (bens materiais e serviços) e processos (organizacionais e de tecnologia relevantes para o desenvolvimento sustentável do Estado); gerar empregos, aumentar a arrecadação do Estado (desenvolvimento econômico e melhoria da qualidade de vida); e fornecer infraestrutura física e tecnológica para o crescimento do Porto.

Finalmente, salienta-se que uma parcela dos empreendimentos vinculados ao cluster PD surgiram de projetos desenvolvidos em Universidades pernambucanas. Esses projetos e empreendimentos já renderam 95 prêmios e reconhecimentos ao PD, além de 79 projetos ligados a inovação (Porto Digital, 2015). 


\subsection{A Interatividade do Caso com o Contexto}

A pesquisa analisa um EC que agrega as estratégias articuladas pelo cluster pernambucano de TIC, denominado Porto Digital. São várias as razões que nos levaram a optar por esta modalidade de EC, especificamente, o tipo de EC naturalista aplicado a um APL (Stake, 2005; 2000). Um aspecto significativo que justifica a sua utilização se deve ao fato de que os EC estão agora estabelecidos como estratégias de pesquisa (Stake, 2005; 2000; 1995) e os equívocos anteriores sobre estudos de casos estão sendo limitada a geração de hipóteses, ao invés da construção de teoria (Flyvbjerg, 2006).

A primeira razão da escolha de um estudo de caso é o acesso, pois, assim existem mais possibilidades de se falar com um leque maior de atores relacionados com o caso estudado. No caso do $\mathrm{PD}$, permitiu-se $\mathrm{o}$ acesso regular aos atores envolvidos $\mathrm{e}$ as suas empresas instaladas nesse cluster. A segunda razão é a de que os clusters que fomentam esses espaços de ação compartilhada são considerados espaços de governança e trocas em redes, nos quais possuem alguns procedimentos estabelecidos por meio dos relacionamentos, tensões e estratégias políticas adotadas. Além disso, pelo fato dos recursos serem limitados de um pesquisador em início de carreira, Stake (1995) recomenda que seja adotada a modalidade de EC único. Em se tratando da análise de um cluster essa escolha se deve ao fato de que a identificação das estratégias negociadas nos arranjos institucionais é fruto de um trabalho extremamente complexo e as fronteiras entre estas não são claramente definidas. Deste modo, a perspectiva do EC naturalista permite ao pesquisador investigar essas ambiguidades dentro de um contexto delimitado da vida contemporânea.

Existe também um número de estudos precedentes que desenvolveram análises interpretativas em torno de EC sobre clusters de TIC, como é o estudo de caso da indústria de Franca elaborado por Assunção Júnior (2006) ou o estudo de caso elaborado por Berbel (2008) que visa compreender como ocorre o processo de internacionalização de um Cluster de empresas de software.

Os resultados gerados nestes exemplos não necessariamente apoiaram o objetivo do presente estudo, que visa compreender como o EC naturalista contribui para o desenvolvimento de pesquisas em clusters de TIC e Economia Criativa. Em geral, esses estudos de caso em clusters permitiram acessar os líderes engajados em assegurar seus processos e as estratégias que são estabelecidas. O cluster de
TIC e Economia Criativa de Pernambuco, denominado Porto Digital, possui uma estratégia sedimentada que demarca uma história particular e um conjunto de identidades a serem estudadas. Estas questões relatadas anteriormente têm sido investigadas como exemplos globais e bemsucedidos de integração de diferentes poderes institucionais, conforme ilustram os trabalhos pioneiros em torno do Vale do Silício, Terceira Itália e Baden-Wurttenburg.

Finalmente, os clusters de TIC constituem a unidade básica de agregação que expressam uma solidariedade orgânica, nos termos descritos por Durkheim (1977), ou seja, a integração dos atores que a compõe é resultado da especialização que demanda interdependência. Clusters de TIC como o Porto Digital, de certa forma confirmam a ênfase de Durkheim (1977) na divisão social do trabalho, uma vez que as ligações entre as pessoas estão baseadas na variedade e complementaridade de suas habilidades.

\subsection{A Equipe de Pesquisadores e as Questões Éticas envolvidas no Estudo}

Este estudo contou com a dedicação de pesquisadores vinculados ao Grupo de Estudos e Pesquisas "Lócus de Investigação em Economia Criativa" da Universidade Federal de Pernambuco (UFPE), especificamente, no Programa de PósGraduação em Administração (Propad/UFPE). O grupo de pesquisa Lócus tem por objetivo "construir conhecimento acerca dos negócios com TIC e da economia criativa, que possam subsidiar ações acadêmicas, organizacionais e sociais, no contexto da sociedade contemporânea" (Propad, 2015, p. 01).

Os pesquisadores que estão envolvidos na investigação do presente estudo estão inseridos na linha de pesquisa do grupo Lócus que visa investigar aspectos relacionados às Indústrias Criativas e os Arranjos Institucionais, temáticas onde o APL estudado encontra-se inserido. Nesse sentido, a referida linha de pesquisa possui o intuito de "investigar as tecnologias de gestão inovadoras e as relações de governança nos arranjos institucionais envolvidos com sistemas que se articulam no âmbito da indústria criativa" (Propad, 2015, p. 01).

As questões éticas permearam o trabalho de campo desta pesquisa, pois, estiveram presentes na forma de acessar as informações, no verdadeiro interesse pelas singularidades e funcionalidades do APL estudado, na forma de expor os indivíduos consultados dentro de limites previamente definidos, além da coerência que foi estabelecida com relação à 
interpretação das informações acessadas referentes ao arranjo (Stake, 2005; 2000; 1998).

\subsection{O método da Observação no Contexto do APL Pernambucano}

Com relação ao método da observação, os estudos de caso naturalistas os apresentam como fundamentais para a compreensão do contexto em estudo. Em se tratando das observações diretas (aproximação com o contexto estudado) e indiretas que este estudo produziu com relação ao APL pernambucano, constatamos alguns aspectos que aqui destacaremos: o primeiro deles é referente à inserção do APL pernambucano em cadeias produtivas; o segundo trata acerca das relações de parcerias que estão sendo promovidas; e o terceiro, referente às redes de negócios e as relações com laços fracos. Todas essas costuras são realizadas a partir de uma unidade de análise específica, espacialmente delimitada, porém expressando múltiplos interesses convergentes e divergentes, permeado por processos interativos e inseparáveis que podem ou não são possíveis generalizações para além do recorte estudado.

\subsubsection{Inserção do APL Pernambucano em Cadeias Produtivas}

Com relação à inserção do PD em cadeias produtivas, observamos em documentos e entrevistas concedidas pelas lideranças desse arranjo que existem articulações para que os artefatos culturais produzidos por esse arranjo dialoguem com as principais demandas das cadeias produtivas do Estado de Pernambuco, conforme corroboram os estudos do CGEE - Centro de Gestão e Estudos Estratégicos (2011) denominado de "Cadeias Produtivas Selecionadas no Recife" e que visou compreender a nova dinâmica socioeconômica e científico-tecnológica do Recife; e o estudo produzido pela consultoria Oremi e Mega (2010) intitulado de "Modelagem Conceitual e Planejamento de Cenários do Pólo de Produção de Conteúdo Cultural de Pernambuco" que teve como objetivo planejar, orientar e criar organizações empresariais de produção de conteúdo criativo.

Essas ações citadas anteriormente e somadas à própria expansão do Porto Digital, consolidada por meio da Lei Municipal do Recife $\mathrm{n}^{\circ} 17.762$, no qual expandiu territorialmente o arranjo, incluindo um quadrilátero no bairro de Santo Amaro e o seu escopo de atuação por meio da criação do equipamento cultural denominado de Portomídia -
Centro de Empreendedorismo e Tecnologia da Economia Criativa, no qual são incorporados os setores criativos de multimídia, games, cinema, design, música e fotografia (Porto Digital, 2015).

A inserção das empresas vinculadas ao cluster Porto Digital em cadeias produtivas importantes para o país, como as de petróleo e gás, estão sendo negociadas a partir da articulação de empreendedores com representantes do Estaleiro Atlântico Sul e da Refinaria de Petróleo General Abreu e Lima, ambos instalados no Complexo Portuário e Industrial de Suape, no Município do Cabo de Santo Agostinho/PE. No trecho da entrevista concedida pelo gestor do NGPD podemos observar esta intenção: [...] "Houve um seminário e a gente vem fazendo isoladamente um conjunto de reuniões com alguns empresários e muito intensamente com o pessoal do Estaleiro e da Refinaria" (Jornal do Commercio, 2011, p. 1).

Assim é perceptível que dentro de suas redes de negócios, o Arranjo Produtivo Local de TIC busca constituir mais conexões para incrementar a produção tanto dos bens simbólicos que produzem quanto dos outros segmentos. Informações dessa natureza, ou seja, que exigem o amadurecimento de dados iniciais para uma posterior avaliação se alcançam por meio do exercício das categorias de observação de campo e interatividade com o contexto inspirados no EC naturalista, como apresentado anteriormente.

Outra liderança vinculada ao Núcleo de Gestão do Porto Digital (NGPD), afirma em entrevista que o setor de TIC ainda está isolado dos demais dos segmentos produtivos. Para ele, poucos são os empresários pernambucanos que recorrem aos serviços do PD para incrementar seus negócios. Por isso, identificou-se que a falta de integração entre os diversos segmentos da economia de Pernambuco com a tecnologia da informação constitui um entrave a ser vencido pelas empresas de TIC que atuam no $\mathrm{PD}$, assim como pelos líderes da economia tradicional que necessitam de aparato tecnológico para alavancar a competitividade de suas empresas (Jornal do Commercio, 2011).

A partir dessas evidencias constata-se que o recorte de estudo de caso pode ajudar na compreensão das lógicas que regem o parque, suas instituições regulatórias, além de auxiliar na captação da natureza dos vínculos entre os agentes e as estruturas. Desse modo revela-se uma escolha acertada para pesquisas que objetivam tratar de questões relacionadas ao empreendedorismo e inovação em arranjos organizacionais que dependem 
da interpretação da das relações entre empresas, estado e sociedade.

\subsubsection{Relações de Parcerias captadas no Campo de pesquisa}

A inserção dos investigadores do Locus no campo de pesquisa estudado permitiu enxergar as parcerias desenvolvidas no Arranjo, que são fatores determinantes para a consolidação do APL de TIC da Cidade do Recife. Além disso, essas interlocuções exercem um importante papel na captação de outros elementos relevantes para geração de inovação como é o caso dos recursos humanos e financeiros. Vejamos um recorte elucidativo de alguns trechos de observações realizadas:

Eu já tenho parceria com todas as empresas, já ligo pra lá dizendo 'oh eu tenho um estagiário'... quase sempre eles entram. Então eles começam a ficar nas empresas estagiando, no estágio supervisionado da universidade e eu supervisiono esse estágio. Mais tarde eles se formam e eles são contratados pelas empresas (Observação 01).

A articulação com organizações de outros locais também se mostrou como um elemento essencial para suprir determinados déficits do setor de TIC, como constatado no recorte de outra observação abaixo:

\begin{abstract}
Não existe ninguém local que tenha o conhecimento que a gente precisa. Porque a gente atua no mundo, a gente atua nos Estados Unidos. A gente não atua em Recife, então você teria que ter outras empresas aqui que estivessem atuando no mesmo mercado e numa condição de liderança ou de conhecimento focado em uma área que a gente não dominasse. Essa realidade hoje, não existe aqui localmente (Observação 02).
\end{abstract}

Essa visão colabora para expandir a perspectiva do EC naturalista ao promover um conhecimento mais aprofundado do foco em análise, o que permite a realização de EC instrumental que permite a extrapolação das fronteiras do caso estudado para melhor entendimento do episódio em estudo, corroborando com Coutinho e Chaves (2002). Para comprovar a contemporaneidade dessa perspectiva de EC, os relatos corroboram com pensamentos de teóricos como Powell e Grodal (2005), Julien (2010), Balestro (2006), Eber e Jarillo (1998) que afirmam que os clusters auxiliam na disseminação da informação, no aprendizado interorganizacional e no compartilhamento e acesso a recursos. Nesse tipo de relacionamentos Stake (2005) nos subsidia na compreensão da natureza das ligações estabelecidas e como se formam as novas configurações organizacionais em rede. Essa abordagem naturalista tem a utilidade de auxiliar no estabelecimento de um ponto referencial a partir do qual se fazem as análises dos ambientes externos e internos da unidade estudada, auxiliando no delineamento das vocações, análise de recursos e competências dos stakeholders e as impressões das lideranças que influenciam nos rumos do aglomerado estudado (Santos, 2017).

\subsubsection{Redes de Negócios e relações com Laços Fracos}

A complexidade dos fenômenos demanda, por exemplo, o enfoque de redes, o que requer metodologias que deem conta de sistemas envolvendo um número elevado de agentes. Essa abordagem se consegue com estudos de caso multicasos que permitem desenvolver uma visão global do fenômeno estudando vários casos. No que se refere ao cluster Porto Digital é uma iniciativa que tem gerado uma série de encadeamentos importantes para se chegar à atual situação em que se encontra o setor de TIC. Outro exemplo é o Centro de Estudos e Sistemas Avançados do Recife (C.E.S.A.R.), uma iniciativa do Centro de Informática da Universidade Federal de Pernambuco (CIn/UFPE) que contribuiu para o fortalecimento do papel de órgãos como Instituto Tecnológico de Pernambuco (ITEP), que faz as mediações entre mercado e universidades.

Os vínculos entre empresas do segmento e as universidades se tornam cada vez mais estreitos, pois, observa-se a procura crescente pelos laços fracos das redes de negócios. Segundo Sérgio Cavalcante, Superintendente do C.E.S.A.R., incubadoras como o C.E.S.A.R são pontes importantes entre a academia universitária e os interesses das empresas.

[...] C.E.S.A.R desenvolve soluções de Engenharia avançada que abrangem todo o processo de geração de inovação em e com TICs - começando com estudos e pesquisas dentro do ambiente do próprio cliente em busca de possibilidades de inovação para o negócio, passando pelo desenvolvimento da ideia, concepção e prototipação e chegando até a execução completa do serviço ou produto" (Jornal do Commercio, 2011, p. 1). 
Fatores que contribuem para essa aproximação são as investidas de políticas públicas, principalmente as impostas pelo Ministério da Ciência, Tecnologia e Inovação (MCTI), a exemplo dos editais que financiam projetos de pesquisa e desenvolvimento a fundo perdido, o que gera uma grande procura de empresários e pesquisadores.

Igualmente, outros dispositivos de desenvolvimento mais facilmente alcançados são as bolsas de desenvolvimento tecnológico e extensão inovadora, porém, fugindo do tradicional interesse que é a produção de artigos científicos, a intenção para esses casos é de direcionar os investimentos para transferências tecnológicas e registro de patentes (bolsa DT). Aqui esse método de EC se mostra indicado para objetos de estudo que tenham como propósito compreender sistemas de inovação, questões relacionadas a atividades empreendedores; políticas públicas de incentivo à inovação tecnológica.

Esses tipos de cenários representam uma aplicação típica em situações atuais de pesquisa utilizando o EC, como mostram Hancock e Algozzine (2016). Compreender a heterogeneidade dessas estruturas e a dinâmica dessas redes exige EC instrumental/multicasos para que a representatividade do fenômeno seja alcançada, a interatividade nas mais variadas situações do negócio seja mapeada, o trabalho experiente e questões éticas de uma equipe de pesquisadores sejam colocados a prova. Esses estudos pedem reflexões teórico-metodológicas autóctones e conectadas a investigações experimentais da dinâmica dos fenômenos estudados.

\section{Considerações Finais}

Os estudos de caso qualitativo auxiliam os pesquisadores no desenvolvimento de seus respectivos estudos. Entretanto, que diversos cuidados devem ser tomados para que esses estudos possam ter critérios de qualidade e confiabilidade bem articulados e permitem acessar as particularidades dos fenômenos estudados.

Em relação ao estudo de caso naturalista o que pode ser apreendido, é que este método de pesquisa busca fornecer informações para que seu leitor possa apreender a partir de suas reflexões sobre o caso apresentado. Ressaltando que o aprendizado de um determinado caso dependerá do conhecimento de outro caso semelhante que o leitor venha a conhecer. Por isso, de forma intuitiva o pesquisador e o leitor poderão determinar se o aprendizado possibilitado pelo estudo de caso poderá ou não ser utilizado para outro caso similar.
$\mathrm{Na}$ perspectiva de EC naturalistas, o conhecimento é construído socialmente. No entanto, uma problemática percebida ao longo do estudo é que para se transmitir coerentemente a experiência adquirida com o caso é necessário que o pesquisador seja capaz de assimilá-lo ao máximo, para que sua transferência ocorra com rigor a sua experiência adquirida. Por isso, corre-se um risco quando o pesquisador, em decorrência do caso ser muito complexo ou extenso, não o conheça profundamente, ou a sua descrição possa está comprometida, onde, o conhecimento possibilitado por meio de sua experiência não seria plenamente possível.

Em referência as pesquisas no campo dos estudos organizacionais, os EC naturalistas auxiliam no entendimento e análise dos mecanismos organizacionais; nas relações estabelecidas ou existentes entre as instituições regulativas, normativas e cultural-cognitivas. Poderão ser estudos que tratem de aspectos ligados à inovação, a aprendizagem e a geração de conhecimentos, pesquisas que tratem das relações entre as organizações, o Estado e a sociedade, em suas diversas situações. Em se tratando de pesquisas que abordem as questões de relacionamentos inter ou intraorganizacionais, Robert Stake orienta no sentido da análise se basear na maneira pela qual os laços entre os atores que constituem essas redes são estabelecidos.

Com relação à análise do caso ilustrativo do APL de TIC e Economia Criativa, denominado Porto Digital, alguns conceitos a partir das apreensões observadas ao longo da questão experiencial foram obtidos na construção do estudo e durante o período de coleta de dados por meio de entrevistas e observação. Neste estudo, o EC do tipo naturalista permitiu, por um lado, um entendimento melhor acerca da forma como se constituiu esse APL, do que ocorre em seu cotidiano, às relações e articulações que se estabelecem entre as redes que estão inseridas e as consequências delas. Todos estes nuances são relevantes para se conhecer o caso ao se desenvolver um olhar fenomenológico, um olhar de dentro, já que o pesquisador imerge e acaba fazendo parte do fenômeno. É este tipo de apreciação que temos interesse em aprofundar ao pautarmos pela lógica dos estudos interpretativos para o desenvolvimento do EC e, desse modo, compreender as percepções dos sujeitos da pesquisa com relação ao Porto Digital.

Sobre a questão da generalização, uma crítica comum ao estudo de caso é dizer que o pesquisador não pode generalizar para além do caso. Uma provável resposta a isso se centra em registrar que cada cluster é único. Nisso, o Porto Digital tem 
um conjunto rico e variado de práticas estratégicas e de governança que se adéquam a investigações empíricas dessa natureza. Nesse sentido, não é possível se demarcar a existência de generalizações estatísticas ou comparar um cluster com outros. No entanto, é possível fazer generalizações analíticas a partir da aplicação das teorias e técnicas em outros clusters.

Com relação às implicações gerenciais e as propostas futuras para formulação de novos estudos, percebemos a necessidade de se produzirem estudos interpretativos que se inspirem na concepção de estudo de caso naturalista, a exemplo do sugerido por Robert Stake, especialmente, pesquisas vinculadas as perspectivas de estudos de casos incorporados no contexto de um arranjo institucional, como no caso do Porto Digital.

\section{Referências}

Abma, T. A.; Stake, R. E. (2014). Science of the particular: An advocacy of naturalistic case study in health research. Qualitative Health Research, v. 24, n. 8, p. 1150-1161.

Aires, L. (2011). Paradigma qualitativo e práticas de investigação educacional. Lisboa: Universidade Aberta.

Assunção Júnior, J. J. C. (2006). Modelo de Evolução de Clusters: Estudo de Caso da indústria calçadista de Franca. Dissertação (mestrado). São Paulo: Escola de Administração de Empresas de São Paulo.

Balestro, M. V. (2006). Capital Social, Aprendizado e Inovação:um estudo comparativo entre redes de inovação na indústria de petróleo e gás no Brasil e Canadá. Tese (Doutorado). Brasília: UnB.

Bauera, M. W.; Gaskell, G. (Ed.). (2002). Pesquisa qualitativa com texto: imagem e som: um manual prático. (trad. de Pedrinho A. Guareschi). Rio de Janeiro: Vozes.

Bell, J. (1989). Doing your research project: a guide for the first-time researchers in education and social science. $2^{\mathrm{a}}$ reimp. Milton Keynes, England: Open University Press, pp. 145.

Berbel, A. C. A. (2008). O Processo de internacionalização de um cluster de empresas de software: O caso do Porto Digital. Dissertação (mestrado). Rio de Janeiro: Universidade Federal do Rio de Janeiro.

Berger, P.; Luckmann, T. (2006). A construção social da realidade. 26. ed. Petrópolis: Vozes.
Biggart, N. W. (1977). The creative-destructive process of organizacional change: the case of the post Office. Administrative Science Quarterly, v. 22, p. 410-426.

Boeira, S. L.; Vieira, P. F. (2006). Estudos organizacionais: dilemas paradigmáticos e abertura interdisciplinar. In: Godoi, C. K.; Bandeira-de-Mello, R.; Silva, A. B. (Org.). Pesquisa qualitativa em estudos organizacionais: paradigmas, estratégias e métodos. São Paulo: Saraiva.

Bryman, A. (1992). Research Methods and Organization Studies. 2nd ed. Contemporary Social Research Series. London \& New York: Routledge. 259 p.

Burrell, G.; Morgan, G. (1979). Sociological paradigms and organisational analysis: Elements of the sociology of corporate life. London: Heinemann.

Célia, M.; Loiola, E. (2014). Aprendendo a aprender: análise de três estudos de caso em aprendizagem organizacional a partir do construtivismo. Organizações \& Sociedade, v. 8, n. 22.

CGEE. (2011). Inovações Tecnológicas e Cadeias Produtivas selecionadas: Oportunidades de negócios para o município de Recife (PE). Brasília: Centro de Gestão e Estudos Estratégicos.

Coutinho, C. P.; Chaves, J. H. (2002). O estudo de caso na investigação em Tecnologia Educativa em Portugal. Revista Portuguesa de Educação, 15(1), pp. 221-243.

Creswell, J. W. (2010). Projeto de pesquisa: métodos qualitativo, quantitativo e misto. 3.ed. Porto Alegre: Artmed/Bookman.

Demo, P. (2001). Pesquisa e informação qualitativa: aportes metodológicos. São Paulo: Papirus.

Denzin, N. K. (2009). The research act: a theoretical introduction to Sociological methods. New Brunswick: Transaction Pub.

Denzin, N. K.; Lincoln, Y. S. (2005). Paradigmatic controversies, contradictions, and emerging confluences. In: Denzin, N. K.; Lincoln, Y. S. (Eds.). The Sage handbook of qualitative research. $3^{\mathrm{a}}$ ed. Thousand Oaks: Sage Publications.

Easterby-Smith, M. (1999). Pesquisa Gerencial em Administração. Uma guia para monografias, dissertações, pesquisas internas e trabalhos em consultoria. São Paulo: Pioneira. 172 p. 
Ebers, M.; Jarillo, J. C. (1998). The construction, forms, and consequences of industry networks. International Studies of Management and Organization, v. 27, p. 3-21, Winter.

Eisenhardt, K. M. (1989). Building theories from case study research. Academy of management review, v. 14 , n. 4 , p. 532-550.

Etzkowits, H.; Leydsorff, L. (1997). Universities in the global knowledge economy: a triple helix of academic-industry-government relations. London: Cassel.

Fidel, R. (1992). The case study method: a case study, In: Glazier, J. D. \& Powell, R. R. Qualitative research in information management. Englewood, CO: Libraries Unlimited, p.37-50.

Flick, U. (2009). Introdução à pesquisa qualitativa (Tradução José Elias Costa). $3^{\mathrm{a}}$ ed. Porto Alegre: Artmed.

Gaskell, G.; Bauer, M. W. (2002). Para uma prestação de contas pública: além da amostra, da fidedignidade e da validade. In: Bauer, M. W.; Gaskell, G. Pesquisa qualitativa com texto, imagem e som: um manual prático. Petrópolis: Vozes.

Gioia, D. A.; Pitre, E. (1990). Multi-paradigm perspectives in theory building. Academy of Management Review, Birmingham, AL, v. 15, n. 4, p. 584-602.

Godoi, C. K.; Bandeira-de-Mello, R.; Silva, A. B. (Org.). (2010). Pesquisa Qualitativa em Estudos Organizacionais: paradigmas, estratégias e métodos. São Paulo: Saraiva.

Godoi, C. K.; Balsini, C. P. V. (2010). A pesquisa qualitativa nos estudos organizacionais brasileiros: uma análise bibliométrica. In: Godoi, C. K.; Bandeira-de-Mello, R.; Silva, A. B. (Org.). Pesquisa Qualitativa em Estudos Organizacionais: paradigmas, estratégias e métodos. São Paulo: Saraiva.

Godoy, A. (1995). A Pesquisa qualitativa - tipos fundamentais. Revista de Administração de Empresas. V.35, n.3, p.20-29.

Godoy, A. S. (1995). Introdução à Pesquisa Qualitativa e suas Possibilidades. Revista de Administração de Empresas. São Paulo, v. 35, n. 2, p. 57-63, Mar./Abr.

Goode, W. J. e Hatt, P. K. (1968). Métodos em pesquisa social. $2^{\mathrm{a}}$ ed. São Paulo: Nacional.

Hancock, D. R.; Algozzine, B. (2016). Doing case study research: A practical guide for beginning researchers. New York \& London: Teachers College Press.

Hartley, J. F. (1995). Case studies in organizational research. In: Cassell, C. e Symon, G. (Ed.). Qualitative methods in organizational research: a practical guide. London: Sage.

Hassard, J. (1991). Multiple Paradigms and Organizational Analysis: A Case Study. Organization Studies, v. 12, n. 2, p. 275-299.

Heracleous, L.; Barrett, M. (2001). Organizational change as discourse: communicative actions and deep structures in the context of information technology implementation. Academy of Management Journal, v. 44, n. 4, p. 755-778.

Hirsch, P. M. (1975). Organizational effectiveness and the institutional environment. Administrative Science Quarterly, v. 20, n. 3, p. 327-44.

Jornal do Commercio. (2011). Inovação em Pernambuco. Caderno Especial. Recife: Jornal do Commercio, jul. 2011.

Julien, Pierre-André. (2010). Empreendedorismo regional: e economia do conhecimento. São Paulo: Saraiva. 399 p.

Langley, A.; Royer, I. (2006). Perspectives on Doing Case Study Research in Organizations. M@n@gement, v. 9, n. 3, p. 81-94.

Lawrence, P. R.; Lorsch, J. W. (1967). Differentiation and integration in complex organizations. Administrative Science Quarterly, v. 12, n. 1, p. 1-47.

Leão, A. L. M. S.; Vieira, R. S. G.; Gaião, B. F. S.; Souza, I. L. (2012). O Que Podemos Aprender com o Estudo de Casos em Administração? Um Ensaio Baseado na Abordagem Naturalista de Robert Stake. AOS. v. 1, n. 1, p. 45-59.

Leão, A. L. M. S., Mello, S. C. B.; Vieira, R. S. G. (2009). O Papel da Teoria no Método de Pesquisa em Administração. Organizações em contexto, Ano 5, n. 10, julho-dezembro.

Lewis, M. W.; Grimes, A. J. (1999). Metatriangulation: Building theory from multiple paradigms. Academy of Management Review, Birmingham, AL, v. 24, n. 4, p. 672-690.

Lincoln, Y. S.; Guba, E. G. (1985). Naturalistic inquiry. London: Sage Publications.

Lima, A. C. C., Maia, G. L. L., Soares, N., \& Ipiranga, A. S. (2002). Uso do método qualitativo em estudos sobre cultura e mudança. Uma análise exploratória da produção cientifica nos Anais do Enanpad. In: CLADEA. Anais...Porto Alegre. 
Mariz, L. A. C., Silva, S. M. G., Dourado, D., \& Regis, H. P. (2005). O reinado dos estudos de caso em Teoria das Organizações: imprecisões e alternativas. Cadernos EBAPE.BR, 3(2), 1-14.

Martinelli, M. L. (1999). Pesquisa Qualitativa: um instigante desafio. São Paulo: Veras Editora.

Mckinsey Global Institute. (2011). Building competitive cities: the key to Latin American growth. Nova York: McKinsey \& Company.

Meirinhos, M.; Osório, A. (2010). O estudo de caso como estratégia de investigação em educação. EduSer - Revista de Educação, v. 2, n. 2.

Merriam, S. B. (1988). Case study research in education. A qualitative approach. San Francisco (CA): Jossey-Bass.

Merriam, S. B. (1998). Qualitative research and case study applications in education. San Francisco (CA): Jossey-Bass.

Oremi e Mega Consultores. (2010). Modelagem Conceitual e Planejamento de Cenários do Polo de Produção de Conteúdo Cultural de Pernambuco (Relatório Final). Recife: Oremi Consultoria, Articulação e Negócios e Mega Consultores Associado.

Paiva Júnior, F. G.; Corrêa, M. I. S.; Souza, A. C. R. (2006). A identidade cultural e a articulação de caráter empreendedor na busca por um desenvolvimento local. In: XXX Encontro da ANPAD. Anais... Salvador: ANPAD.

Paiva Júnior, F. G.; Leão, A. L. M. S.; Mello, S. C. B. (2011). Validade e Confiabilidade na Pesquisa Qualitativa em Administração. Revista de Ciências da Administração. V. 13, n. 31, p. 190209, set./dez.

Paiva Júnior, F. G.; Mello, S. C. B. (2008). Pesquisa Qualitativa nos Estudos Organizacionais: Contribuições Fenomenológicas de Alfred Schütz. Organizações em contexto, Ano 4, n. 8, dezembro.

Pinto, M. R.; Santos, L. L. S. (2008). Em Busca de uma Trilha Interpretativista para a Pesquisa do Consumidor: Uma Proposta Baseada na Fenomenologia, na Etnografia e na Grounded Theory. RAE-eletrônica. v. 7, n. 2, Art. 25, jul./dez.

Porto Digital. (2010). Perfil Geral do Porto Digital. Recife: Porto Digital.

Porto Digital. (2015). Página Institucional do Porto Digital. Recife: Porto Digital. Disponível em: <http://www.portodigital.org.br>. Acesso em 03 jun. 2015.

Powell, W.; Grodal, S. (2005). Networs of innovators. In: Fagerberg, J. et al. The Oxford Handbook of Innovation. Oxford: Oxford University Press. P. 56-85.

Propad. (2015). Lócus de Investigação em Economia Criativa. Recife, UFPE.

Rocha, R. A.; Ceretta, P. S. (1998). Pesquisa qualitativa: um desafio à ciência social. In: XXII Enanpad, Anais... Foz do Iguaçu: ANPAD.

Roesch, S. M. A. (2009). Casos para ensino em Administração: notas sobre a construção de casos para ensino. Revista de Administração Contemporânea, vol. 11(2), p.213-34, abr-jun.

Santos, B. S. (2006). Um discurso sobre as ciências. $4^{\mathrm{a}}$ ed. São Paulo: Cortez.

Santos, E. L. (2017). The scientific field of administration: an analysis from the circle of theoretical matrices. Cadernos EBAPE.BR, v. 15, n. 2 , p. $209-228$.

Sebring, R. H. (1977). The five million dollar misunderstanding: a perspective on state government-university interoganizational conflicts. In: Administrative Science Quarterly, v. 22 , n. 4, p. 505-23.

Senger, I.; Paço-Cunha, E.; Senger, C. M. (2013). O estudo de caso como estratégia metodológica de pesquisas científicas em administração: um roteiro para o estudo metodológico. Revista de Administração, v. 3, n. 4, p. p. 93-116.

Silva, A. B. D.; Neto, J. R. (2010). Perspectiva multiparadigmática nos estudos organizacionais. In: Godoi, C. K. et al. (Ed.). Pesquisa Qualitativa em Estudos Organizacionais: Paradigmas, Estratégias e Métodos. São Paulo: Saraiva. P. 53-87.

Silva, A. D. F. O processo regulador na $[\mathrm{Re}]$ conversão de um Arranjo Produtivo Local: o caso do Portomídia - Centro de Empreendedorismo e Tecnologia da Economia Criativa. Dissertação (Mestrado). Recife: Universidade Federal de Pernambuco.

Silva, J. S.; Fernandes, N. C. M.; Silva, A. D. F.; Paiva Júnior, F. G. (2012). A influência das Redes Interorganizacionais no Processo de Inovação de Jogos Eletrônicos: O Caso do Setor de Games no Porto Digital. In: XXVII Simpósio de Gestão da Inovação Tecnológica da ANPAD, Anais... Salvador. 
Stake, R. E.; Easley, J. A. (1979). Case studies in Science Education. Urbana: University of Illinois.

Stake, R. E. (1988). Case study methods in educational research: Seeking sweet water. In: Jaeger, R. M. (Ed.). Complementary methods for research in education. Washington, DC: American Educational Research Association. P. 253-265.

Stake, R. E. (2000). Case studies. In: Denzin, N. K.; Lincoln, Y. S. (Ed.). Handbook of qualitative research. $2^{\mathrm{a}} \mathrm{Ed}$. Thousand Oaks (CA): Sage.

Stake, R. E. (2005). Qualitative Case studies. In: Denzin, N. K.; Lincoln, Y. S. (Eds.) The Sage handbook of qualitative research. London: Sage Publications.

Stake, R. E. (2006). Multiple case study analysis. New York: The Guilford Press.
Schultz, M.; Hatch, M. J. (1996). Living with Multiple Paradigms: The Case of Paradigm Interplay in Organizational Culture Studies. Academy of Management Review, Birmingham, AL, v. 21, n. 2, p. 529-557.

Schwandt, T. A. (2006). Três posturas epistemológicas para a investigação qualitativa: interpretativismo, hermenêutica e construcionismo social. In: Denzin, N. K.; Lincoln, Y. S. O planejamento da pesquisa qualitativa: teorias e abordagens. 2. ed, Porto Alegre: Bookman.

Silverman, D. (2009). Interpretação de dados qualitativos: métodos para análise de entrevistas, textos e interação. Porto Alegre: Artmed.

Yin, R. K. (1993). Applications of case study research. Thousand Oaks (CA): Sage.

Yin, R. K. (2005). Estudo de Caso: planejamento e métodos. $3^{\mathrm{a}}$ ed. Porto Alegre: Bookman.

\title{
The Naturalistic Case Study in the investigation of Local Productive Arrangements (APL) of Information and Communication Technologies (ICT)
}

\begin{abstract}
The Case Study (EC) involves a divergent conceptual scenario and can be used in diverse research forms in the field of organizational studies with respect to the value and plausibility of the application of this method of investigation in Local Productive Arrangements (APL). In this theoretical-methodological essay, we discuss the conceptual interpellation of the naturalistic EC of Robert Stake amid its possibilities and limitations of use, illustrated through the analysis of the Local Productive Arrangement (APL) of Information and Communication Technologies (ICT) of the State of Pernambuco (Brazil), entitled Porto Digital. By moving from an approach based on the logic of Yin's (2005) functionalist explanation for the treatment of Stake (2005), this view of naturalistic case study represents the opportunity for the development of the interpretive studies that enable the increase of the related knowledge to cooperative business environments contemplating new ICTs.
\end{abstract}

Keywords: Qualitative Methodological Approaches; Case Studies; Local Productive Arrangements, Information and Communication Technology (ICT).

\section{El Estudio de Caso Naturalista en la investigación de Arreglos Productivos locales (APL) de Tecnologías de Información y Comunicación (TIC)}

\section{Resumen}

El Estudio de Caso (EC) involucra un escenario conceptual diverso que puede ser utilizado en formas de investigación diversificadas en el campo de los estudios organizacionales con respecto al valor y la 
plausibilidad de la aplicación de ese método de investigación en Arreglos Productivos locales (APL). En este ensayo teórico-metodológico, discutimos la interpelación conceptual del EC naturalista de Robert Stake en medio de sus posibilidades y limitaciones de uso, ilustradas por el análisis del Arreglo Productivo Local (APL) de Tecnologías de la Información y la Comunicación (TIC's) de Pernambuco (Brasil), denominado Porto Digital. Al migrar de un enfoque fundamentado en la lógica de la explicación de perspectiva funcionalista de Yin (2005) para el tratamiento de Stake (2005), esa visión de estudio de caso naturalista representa la oportunidad para el desarrollo de estudios interpretativos que posibilitan el incremento del conocimiento relacionado con los entornos de negocios cooperativos contemplando las nuevas TIC.

Palabras-Clave: Enfoques Metodológicos Cualitativos; Estudio de caso; Arreglos Productivos Locales; Tecnologías de la Información y la Comunicación (TIC's).

\section{Sobre os Autores}

\section{Anderson Diego Farias da Silva}

Doutorando em Administração pela Universidade Federal de Pernambuco (UFPE) e Visiting PhD Student pela École des Hautes Études Commerciales de Montréal (HEC Montréal - Canadá).

E-mail: andersondiego6@gmail.com

\section{Nelson da Cruz Monteiro Fernandes}

Doutor em Administração pela Universidade Federal de Pernambuco (UFPE)

Professor do Centro Acadêmico do Agreste (CAA), Universidade Federal de Pernambuco (UFPE)

Rodovia BR-104, Km 59, s/n - Nova Caruaru - Caruaru - PE - Brasil - CEP 55002-970.

E-mail: cruzfernandes55@gmail.com

\section{Fernando Gomes de Paiva Júnior}

Doutor em Administração pela Universidade Federal de Pernambuco (UFPE)

Professor do Departamento de Ciências Administrativas (DCA), Universidade Federal de Pernambuco (UFPE)

Centro de Ciências Sociais Aplicadas, Avenida dos Economistas, S/N - Cidade Universitária - Recife - PE - Brasil CEP 50.670-901

E-mail: fernando.paivajr@gmail.com 